\title{
PROPRIEDADES DO PAPEL KRAFT A PARTIR DA MADEIRA JUVENIL DE Pinus maximinoi H.E. Moore E Pinus taeda L.
}

\author{
Umberto Klock $^{1}$ \\ Alan Sulato de Andrade ${ }^{2}$ \\ Eduardo Bittencourt ${ }^{3}$ \\ Ezequiel Zatoni Mocelin ${ }^{4}$ \\ Cristiane Crepaldi ${ }^{5}$
}

\begin{abstract}
RESUMO
A qualidade das fibras da madeira juvenil de Pinus maximinoi H. E. Moore comparando com Pinus taeda L. foi avaliada em material proveniente de plantações de 11 anos de idade localizados no município de Ventania, Estado do Paraná. Foram investigadas 15 árvores por espécie. O processo utilizado para os cozimentos experimentais foi o sulfato (Kraft). O material utilizado para a obtenção de celulose foi obtido de discos coletados na altura do DAP $(1,30 \mathrm{~m})$. As condições experimentais dos cozimentos foram: $18 \%$ de álcali ativo e $25 \%$ de sulfidez; número Kappa de 30 e fator $\mathrm{H}$ total de 1150 , para três repetições para cada espécie. Os cozimentos foram realizados com $1000 \mathrm{~g}$ de cavacos, base seca. Para a confecção do papel a mão em laboratório a celulose foi submetida a tratamentos de refinação em moinho tipo Jokro a $150 \mathrm{rpm}$ por 20, 40 e 60 minutos além do tratamento sem refinação. Os rendimentos brutos médios foram superiores a $45 \%$. Os resultados médios das resistências do papel por tratamento de refinação entre as espécies mostraram tendência semelhante entre ambas, ocorrendo, entretanto, no tratamento sem refinação maior densidade aparente para o papel de Pinus maximinoi. Porém com a aplicação dos tratamentos de refinação o comportamento da densidade aparente tornouse similar. No tratamento sem refinação o valor médio do comprimento de auto-ruptura do papel de Pinus maximinoi foi $11,5 \%$ inferior ao de Pinus taeda, sendo, entretanto, incrementado com o aumento dos tempos de refinação. Com 20 minutos foi ligeiramente superior e aos 40 minutos a diferença é 6,6\% superior, chegando a $7,9 \%$ aos 60 minutos de refinação. A resistência ao estouro no tratamento sem refinação e a 20 minutos apresentou valores inferiores para o Pinus maximinoi. Aos 40 minutos os valores médios são similares, invertendo-se tais valores aos 60 minutos de refinação, quando o estouro é superior para a celulose de Pinus maximinoi. Os valores médios da resistência ao rasgo foram ligeiramente superiores para a celulose de Pinus taeda, porém somente com 40 minutos de refinação houve diferença significativa. A resistência ao estouro e a resistência à tração da celulose e papel de Pinus maximinoi desenvolveu-se de forma mais acentuada com os tratamentos de refinação aplicados. Conclui-se que a madeira de Pinus maximinoi apresenta potencial para a produção de celulose e papel.

Palavras Chave: Cozimento Kraft, Papel feito-a-mão, Fibras de madeira juvenil
\end{abstract}

\section{KRAFT PAPER PROPERTIES FROM Pinus maximinoi H.E. Moore AND Pinus taeda L. JUVENILE} WOOD

\begin{abstract}
This research objective was to evaluate the juvenile wood fiber quality of Pinus maximinoi H. E. Moore compared to Pinus taeda L. The material was sampled from 15 trees with 11 years old from fast grown plantations located at Ventania municipality, Paraná State. Sulfate (Kraft) pulping process was used. The pulping material was from BHD $(1.30 \mathrm{~m})$ disks, cooking conditions were $18 \%$ active alkali, $25 \%$ sulfidity, Kappa number of 30 and $\mathrm{H}$ factor of 1,150 for three repetitions for each specie. Experimental cookings were conduted with $1,000 \mathrm{~g}$ dry basis wood chips. For pulp handsheets evaluation four treatments were considered: without beating, 20, 40 and 60 minutes beating in Jokro type mill at 150 revolutions per minute. The average pulp yield was slightly higher than $45 \%$ for both species, without statistic difference. Without beating treatment $P$. maximino $i$ handsheets showed higher apparent specific gravity, however it became similar to $P$. taeda sheets with beating. Average Tear Index, Burst Index and Breaking Length, showed no statistic difference between species, but
\end{abstract}

\footnotetext{
klockuer@ufpr.br - Doutor Prof. Adjunto do Depto. de Eng. e Tecnologia Florestal da UFPR

2 asulato@floresta.ufpr.br - Mestrando do Curso de Pós-Graduação em Engenharia Florestal da UFPR

3 edubitt@bol.com.br - Mestrando do Curso de Pós-Graduação em Engenharia Florestal da UFPR

mocelin@floresta.ufpr.br - Mestrando do Curso de Pós-Graduação em Engenharia Florestal da UFPR

5 criscrepaldi@yahoo.com.br - Mestranda - Departamento de Madera, Celulosa y Papel - UDG

Recebido para publicação: 16/07/2003

Aceito para publicação: 19/11/2003
} 
considering beating treatments, differences appear in Burst Index and Breaking Length, P. maximinoi pulp showed a better evolution with increasing beating times, at 60 minutes beating treatment $P$. maximinoi sheets showed 7,9\% higher Breaking Length average value. For Burst Index, P. maximinoi sheets showed better improvement with increasing beating treatments, the average value became higher at 60 minutes. The Tear Index decrease with beating, and $P$. taeda pulp showed slightly higher values, but only at 40 beating minutes was a significant difference. From the observed average results it can be concluded that Pinus maximinoi has potential for utilization for pulp and paper.

Keywords: Kraft pulping, Handsheets, Juvenile wood fibers

\section{INTRODUÇÃO}

A madeira de Pinus spp. é essencial ao setor de celulose e papel de fibra longa, que participou com $16,7 \%$ da produção nacional de 9 milhões de toneladas em 2003; e que apresentou até julho de 2004 um crescimento de $1,6 \%$ em relação ao mesmo período de 2003, BRACELPA (1). A expansão do setor prevê aumento na produção de pastas de alto rendimento e celulose Kraft de fibras longas durante a próxima década. Por esta razão é essencial o estudo de novas matérias-primas para o fornecimento de fibras longas de qualidade e o suprimento da necessidade industrial a um ritmo cada vez mais imponente.

Constatado o potencial crescente da madeira de Pinus spp., a necessidade do aprofundamento nos conhecimentos sobre a qualidade da madeira e o resultado observado da espécie Pinus maximinoi - espécie ainda desconhecida no mercado madeireiro e papeleiro, plantada em testes de progênies (PROGRAMA CAMCORE - Central América and México Coniferous Resources) com notável desenvolvimento, vigor e forma em apenas 11 anos, tornando-se importante e oportuno obter-se mais informações sobre a qualidade e variabilidade da madeira produzida pela espécie, visando-se subsidiar as decisões de seleção e melhoramento genético, que, aliados a técnicas silviculturais, poderão definir as características tecnológicas desejáveis para a madeira.

Considerando-se que a proporção de madeira juvenil nas árvores de coníferas, oriundas de florestas de rápido crescimento e de curta rotação, é consideravelmente alta, torna-se fundamental o conhecimento das propriedades para a sua utilização de forma adequada. Em razão destes aspectos, definiu-se como objetivos do estudo avaliar o comportamento das fibras de Pinus maximinoi
H. E. Moore durante o cozimento e no papel, comparando-se as fibras de Pinus taeda L., espécie mais utilizada no País na produção de celulose e papel de fibra longa, cobrindo aspectos das propriedades das fibras para a industrialização de celulose e papel; informações que são básicas na definição do uso final da madeira.

\section{REVISÃO BIBLIOGRÁFICA}

Há poucas informações publicadas disponíveis acerca das propriedades da celulose e do papel proveniente da madeira de Pinus maximinoi. Porém, baseando-se na literatura disponível, é possível fazer comparações com resultados obtidos para árvores de idades semelhantes, isto é, de madeira juvenil, embora crescendo em países como a África do Sul e Colômbia, com certas diferenças metodológicas, permitindo traçar-se preliminarmente um paralelo entre as características apresentadas pela celulose e papel nesse estudo.

Wright \& Wessels (2), obtiveram em seu estudo com Pinus pseudotrobus e Pinus maximinoi, a partir de 10 árvores por espécie, com 10,5 anos de idade, de testes de procedência na África do Sul, rendimentos médios de $45 \%$ em celulose obtida em laboratório pelo processo Kraft, com número Kappa médio de 55. Os resultados médios de resistência do papel de Pinus maximinoi, para tratamentos sem refinação, e com refinação a 2250 - 9000 e 18000 revoluções em moinho tipo $P F I$, variaram a partir do tratamento sem refinação para o índice de estouro de 4,3 a 7,1 $\mathrm{kPa} \cdot \mathrm{m}^{2} \cdot \mathrm{g}^{-1}$; índice de rasgo de 13,5 a 7,9 $\mathrm{mN} \cdot \mathrm{m}^{2} \cdot \mathrm{g}^{-1}$; e comprimento de auto-ruptura de 5,7 a $9,7 \mathrm{~km}$. Os autores concluíram que o Pinus maximinoi apresentou potencial como matéria-prima para celulose quando comparado a outras espécies de Pinus spp, como Pinus pseudotrobus e Pinus patula, embora apresentando menor índice de rasgo. 
Num trabalho posterior, Wright \& Baylis (3), utilizando material de Pinus maximinoi coletado em dois testes de progênies da África do Sul, de 5 árvores com características dominantes com 11 anos de idade, obtiveram resultados similares entre os testes de progênies, com valores médios de rendimento em celulose de $42,3 \%$ e número Kappa de 28. Obtiveram para o comprimento de auto-ruptura $6,16 \mathrm{~km}$, índice de estouro de 4,2 $\mathrm{kPa} \cdot \mathrm{m}^{2} \cdot \mathrm{g}^{-1}$ e índice de rasgo de 12,8 $\mathrm{mN} \cdot \mathrm{m}^{2} \cdot \mathrm{g}^{-1}$, para folhas de papel feitas a mão sem tratamento de refinação.

Ressalta-se que a celulose foi obtida por processo Kraft em micro cozimentos e os cavacos confeccionados a partir de rolos de incrementos retirados a altura do DAP. Os autores concluíram que a madeira de Pinus maximinoi apresentou resultados em celulose e nas propriedades do papel aceitáveis e recomendaram a espécie para plantio em larga escala na África do Sul.

Wright et al. (4), em estudo mais recente com espécies tropicais do gênero Pinus, entre as quais Pinus maximinoi, com árvores de 8 anos de idade e livre de defeitos, plantadas na Colômbia, obtiveram rendimento bruto de aproximadamente $50 \%$ para número Kappa de 50, e 47\% para números Kappa de 38 e 28. Os índices de estouro variaram de 3 a $8 \mathrm{kPa} \cdot \mathrm{m}^{2} \cdot \mathrm{g}^{-1}$, e de 18 a $10 \mathrm{mN} \cdot \mathrm{m}^{2} \cdot \mathrm{g}^{-1}$ para o rasgo, sendo o primeiro valor referente ao tratamento sem refinação. Os autores concluíram que os valores do índice de estouro, encontram-se dentro da faixa normal esperada para celulose não branqueada de espécies de Pinus, que situam-se entre 6 a 9 $\mathrm{kPa} \cdot \mathrm{m}^{2} \cdot \mathrm{g}^{-1}$, já os valores determinados para o índice de rasgo para Pinus maximinoi, bem como das outras espécies estudadas, foram considerados baixos, comparados aos esperados para espécies de Pinus, porém lembram os autores que para árvores de baixa idade, os resultados são compatíveis aos esperados e obtidos por outras espécies do gênero nesta faixa de idade, observando que $o$ aumento da idade deve incrementar também as propriedades ao rasgo em razão da diferenciação dos traqueóides e do aumento da proporção de madeira adulta.

Celulose obtida em laboratório em condições similares a deste trabalho por Foelkel (5), com diferença apenas na porcentagem de álcali-ativo que foi de 19\%, para as espécies: Araucaria angustifolia de idade indeterminada; Pinus elliottii com 8 anos; Pinus taeda com 11 anos; Pinus caribaea var. hondurensis com 8 anos - todas plantadas no Brasil, conjuntamente com Pinus elliottii de 18 anos e Pinus taeda de 16 anos essas plantadas nos Estados Unidos, relatou resultados, em rendimento bruto, para a celulose obtida de madeiras brasileiras variando de 47,2 a $51,84 \%$, sendo superiores à celulose obtida da madeira americana que foi de $43,3 \%$ para Pinus taeda e 45,6\% para Pinus elliottii.

Observa-se que considerados os intervalos nos tempos de refinação correspondentes a densidade de $0,600 \mathrm{~g}_{\mathrm{cm}} \mathrm{cm}^{-3}$, os valores médios similares para o comprimento de auto-ruptura para as espécies plantadas no Brasil, exceto para Pinus caribaea e as duas espécies plantadas nos Estados Unidos que apresentaram valores médios bem superiores.

Watson \& Dadsweel (6), estudando a influência da porcentagem de lenho tardio nas propriedades da celulose, determinaram para Pinus taeda, de 23 anos, com porcentagem de lenho tardio em torno de $38 \%$, valores médios para os índices de rasgo de 276 e 176, estouro de 43 e 75 e comprimento de auto-ruptura de 4,4 a $8,1 \mathrm{~km}$, sendo o primeiro valor correspondente a tratamento sem refinação e o segundo a refinação a 9000 revoluções em moinho tipo Lampen. Os autores concluíram no estudo, que o lenho inicial e lenho tardio produzem celulose com propriedades diferentes, o lenho tardio produz celulose com alto valor na resistência ao rasgo, mas com relativos baixos valores em estouro e tração, enquanto que o lenho inicial produz papel com boa conformação, com resistências mais altas em estouro e tração.

Klock et al. (7) obtiveram valores médios superiores para o papel feito a mão, a partir de celulose obtida em laboratório nas mesmas condições de cozimento, de árvores com 25 anos de Pinus taeda, de diferentes classes diamétricas, para tempos de refinação de 10,20 e 40 minutos. O índice de rasgo variou nas classes diamétricas de 29,6 a 16,9 $\mathrm{mN} \cdot \mathrm{m}^{2} \cdot \mathrm{g}^{-1}$, o índice de estouro de 3,7 a 6,3 $\mathrm{kPa} \cdot \mathrm{m}^{2} \cdot \mathrm{g}^{-1}$ e o comprimento de auto-ruptura de 5,45 a $9,20 \mathrm{~km}$, sendo o primeiro valor 
correspondente ao tratamento de refinação de dez minutos.

\section{MATERIAL E MÉTODOS}

O material para este estudo foi obtido de 15 árvores de Pinus maximinoi H. E. Moore e 15 árvores de Pinus taeda L., de mesma classe diamétrica, provenientes de plantios com 11 anos de idade, localizados na Fazenda Moquém, de propriedade da PISA - Papel de Imprensa S. A., localizada no município de Ventania, Estado do Paraná. A espécie Pinus maximinoi foi escolhida para o estudo por se destacar pelo ritmo e vigor de crescimento na região e pela necessidade de se conhecer a qualidade da madeira produzida pela espécie.

As árvores de Pinus maximinoi foram oriundas de testes de progênies implantados em 1988 pelo programa CAMCORE, sendo representantes das famílias de melhor desenvolvimento em diâmetro, altura e forma.
A espécie Pinus taeda, por sua vez, foi escolhida por ser uma das espécies do gênero Pinus mais plantadas e utilizadas industrialmente no País; e especificamente pela plantação estar localizada em talhão contíguo ao teste de progênie de Pinus maximinoi, permitindo um relacionamento bem estreito das espécies, pois estão em um mesmo sítio.

\section{Amostragem e Preparação do Material}

Foram coletados discos de madeira da posição 1,30m (DAP) com aproximadamente $5 \mathrm{~cm}$ de espessura, de 15 árvores por espécie. A obtenção de celulose pelo processo sulfato ou Kraft e propriedades das fibras em papel feito a mão da madeira dessas espécies foi realizada com cavacos homogeneizados obtidos dos discos por espécie, realizando-se três repetições por espécie.

\section{Produção da Celulose Kraft}

As condições experimentais de cozimento que são apresentadas na tabela 1 .

Tabela 1: Condições experimentais dos cozimentos utilizados

Table 1: Pulping experimental conditions

\begin{tabular}{cc}
\hline Variáveis & Condições \\
\hline Álcali ativo & $18 \%$ \\
Sulfidez & $25 \%$ \\
Relação licor:madeira & $4: 1$ \\
Fator H & 1150 \\
$\mathrm{~N}^{\mathrm{o}}$ Kappa & 30 \\
Temperatura máxima & $170^{\circ} \mathrm{C}$ \\
Quantidade de madeira seca & $1000 \mathrm{~g}$ \\
\hline
\end{tabular}

Com o material obtido foram determinados o rendimento bruto, o teor de rejeitos e o número Kappa.

\section{Confecção do Papel a Partir da Celulose} Obtida de Pinus maximinoi e de Pinus taeda

Para a formação de folhas a celulose foi refinada em moinho tipo JOKRO segundo a norma ABNT 14346, em 3 tratamentos: 20, 40 e 60 minutos, além do tratamento testemunha (sem refinação).
Os ensaios físicos e mecânicos foram realizados de acordo com as recomendações técnicas da ABTCP (8), para gramatura, densidade aparente, resistência ao rasgo, resistência ao estouro e resistência à tração.

\section{RESULTADOS E DISCUSSÕES}

Os resultados médios obtidos para as características da celulose produzida em laboratório pelo processo Kraft, com a madeira de Pinus maximinoi e Pinus taeda, são apresentados na tabela 2 . 
Tabela 2: Rendimento médio bruto, teor de rejeitos e número Kappa médio das celuloses obtidas em laboratório pelo processo Kraft, da madeira de 15 árvores de Pinus maximinoi e de Pinus taeda

Table2: Average yieldings, rejects and kappa numbers from Kraft pulps obtained in laboratory from Pinus maximinoi and Pinus taeda wood of 15 trees from each specie

\begin{tabular}{ccccccc}
\hline Espécie & $\begin{array}{c}\text { Rendimento Bruto } \\
\%\end{array}$ & DP* & DP & Número Kappa & DP \\
& $\%$ & & Teor de Rejeitos $\%$ & & & \\
\hline Pinus maximinoi & 45,26 & 0,25 & 0,20 & 0,10 & 31,74 & 0,43 \\
\hline Pinus taeda & 45,76 & 0,54 & 0,81 & 0,12 & 32,56 & 0,71 \\
\hline $\mathrm{DP}-$ desvio padrão & & & & &
\end{tabular}

Observa-se que os rendimentos médios obtidos nos cozimentos experimentais foram semelhantes entre as duas espécies, e com valores normais para coníferas quando tratadas pelo processo Kraft, cuja faixa de variação em rendimento, dependendo das condições varia entre 40 e $50 \%$, SMOOK (9).

Os números Kappa médios foram ligeiramente superiores aos propostos porém, variaram dentro dos limites aceitáveis na experimentação e, os teores de rejeitos, foram bastante baixos, demonstrando que as duas espécies responderam bem as condições de cozimento, embora estatisticamente a diferença tenha sido significativa a $95 \%$.

A tabela 3 apresenta os resultados médios das propriedades de resistência mecânica e densidade aparente determinados para as folhas de papel feitas a mão, por tratamentos de refinação.

As figuras 1 e 2 ilustram graficamente as evoluções do comportamento médio do Índice de Rasgo, do Índice de Estouro, e do Comprimento médio de Auto-ruptura do papel feito a mão, a partir da celulose Kraft obtido da madeira juvenil das duas espécies estudadas.

Tabela 3: Propriedades das folhas de papel feitas a mão, a partir da celulose Kraft obtida em laboratório da madeira juvenil de 15 árvores de Pinus maximinoi e de Pinus taeda

Table3: Handsheets properties from Kraft pulps obtained in laboratory from Pinus maximinoi and Pinus taeda wood of 15 trees from each specie

\begin{tabular}{cccccc}
\hline Tratamento & $\begin{array}{c}\text { Gramatura } \\
\left(\mathrm{g} \cdot \mathrm{m}^{-2}\right)\end{array}$ & $\begin{array}{c}\text { Índice de Rasgo } \\
\left(\mathrm{mN} \cdot \mathrm{m}^{2} \cdot \mathrm{g}^{-1}\right)\end{array}$ & $\begin{array}{c}\text { Índice de Estouro } \\
\left(\mathrm{kPa} \cdot \mathrm{m}^{2} \cdot \mathrm{g}^{-1}\right)\end{array}$ & $\begin{array}{c}\text { Comprimento } \\
\text { Auto-ruptura } \\
(\mathrm{km})\end{array}$ & $\begin{array}{c}\text { Densidade aparente } \\
\left(\mathrm{g} \cdot \mathrm{cm}^{-3}\right)\end{array}$ \\
\hline Pinus maximinoi & & & & 0,416 \\
\hline Sem Refinação & 62,2 & 22,96 & 1,83 & 3,21 & 0,586 \\
20 minutos & 60,7 & 19,58 & 3,78 & 6,05 & 0,630 \\
40 minutos & 59,1 & 13,68 & 4,22 & 6,83 & 0,686 \\
\hline 6 minutos & 58,2 & 11,21 & 4,23 & 6,98 & 0,387 \\
\hline Pinus taeda & & & & 3,63 & 0,588 \\
\hline Sem Refinação & 61,6 & 23,96 & 2,13 & 5,96 & 0,622 \\
20 minutos & 60,1 & 19,76 & 4,01 & 6,41 & 0,663 \\
\hline 40 minutos & 57,9 & 15,88 & 4,20 & 6,47 & 4,00 \\
60 minutos & 56,9 & 12,31 & & & \\
\hline
\end{tabular}

Obs.: Desvios padrões entre as repetições variaram abaixo dos requerimentos das normas técnicas, entre $0,2 \mathrm{e}$ $1,1 \%$.

* Refinação em moinho laboratorial Jokro a 150 rpm. 


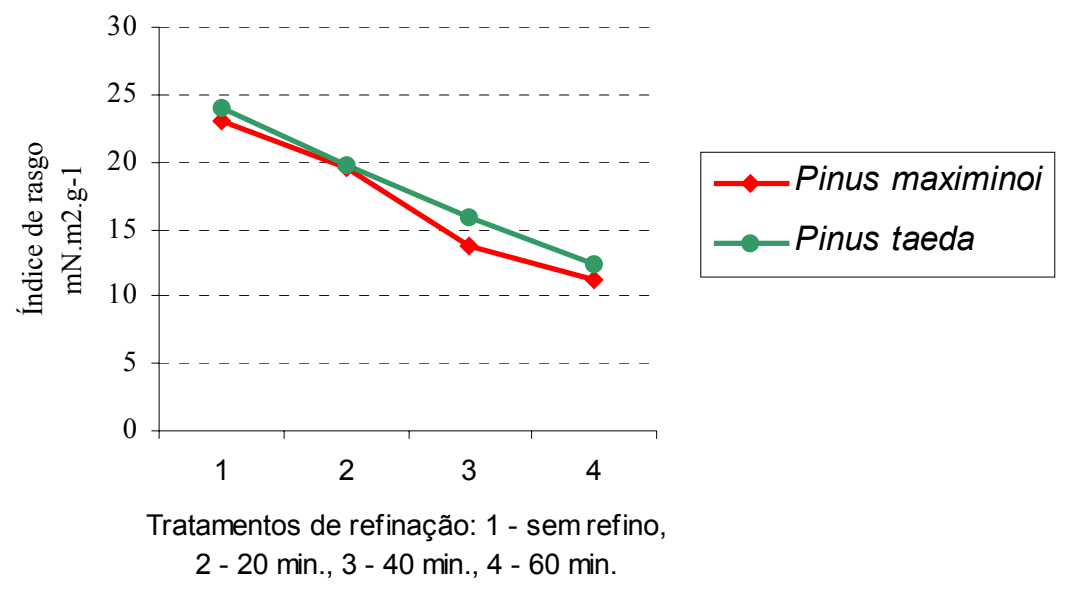

Figura 1: Evolução do Índice de rasgo médio do papel feito a mão em função do tempo de refinação da celulose Kraft da madeira juvenil de 15 árvores de Pinus maximinoi e de Pinus taeda

Figure 1: Average Tear Index evolution from paper handsheets properties from Kraft pulps obtained in laboratory from Pinus maximinoi and Pinus taeda juvenile wood of 15 trees from each specie in function of beating time

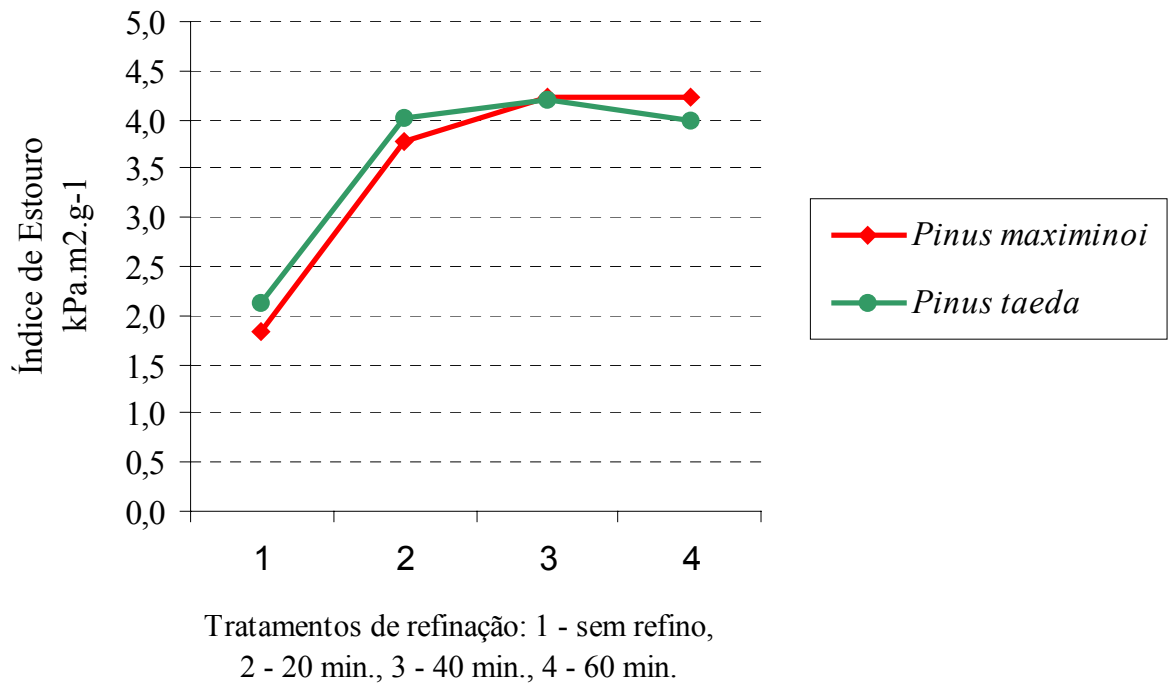

Figura 2: Evolução do Índice de estouro médio do papel feito a mão em função do tempo de refinação da celulose Kraft da madeira juvenil de 15 árvores de Pinus maximinoi e de Pinus taeda

Figure 2: Average Bursting Index evolution from paper handsheets properties from Kraft pulps obtained in laboratory from Pinus maximinoi and Pinus taeda juvenile wood of 15 trees from each specie in function of beating time 


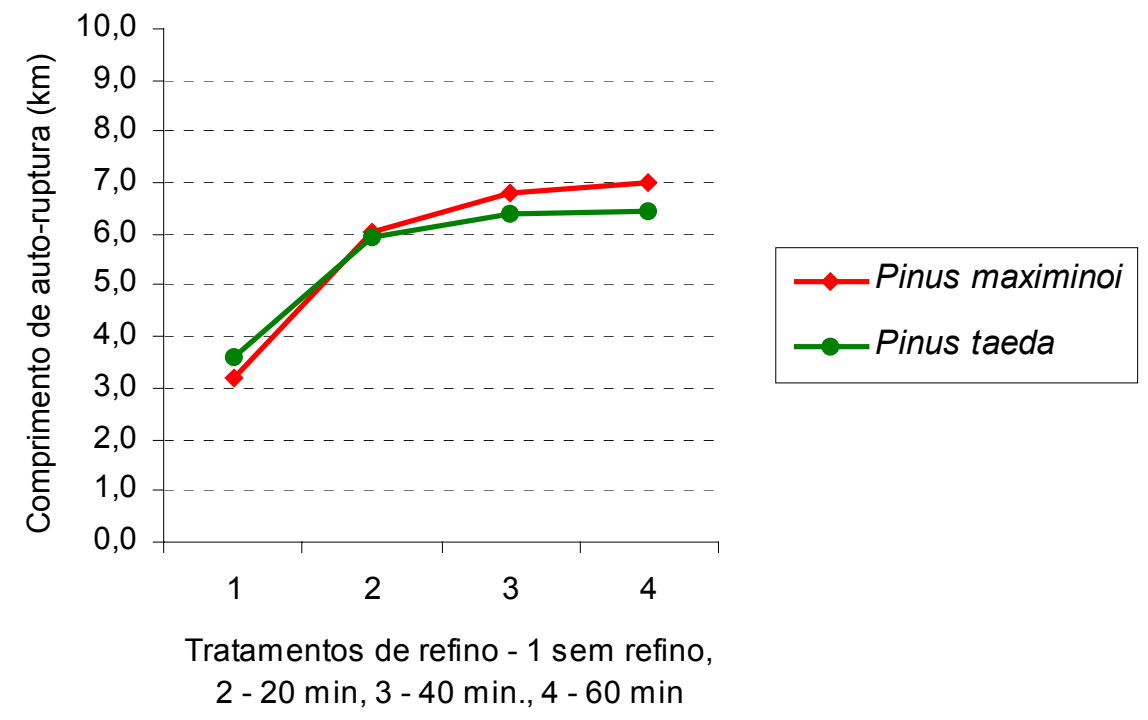

Figura 3: Evolução do Comprimento de Auto-ruptura médio do papel feito a mão em função do tempo de refinação da celulose Kraft da madeira juvenil de 15 árvores de Pinus maximinoi e de Pinus taeda

Figure 3: Average Breaking Strength evolution from paper handsheets properties from Kraft pulps obtained in laboratory from Pinus maximinoi and Pinus taeda juvenile wood of 15 trees from each specie in function of beating time

Observa-se na tabela 3, que a gramatura decresceu com os tratamentos de refinação, em razão da formação de partículas muito finas, que ocorre no processo de refinação das fibras (traqueóides), e são perdidas na formação das folhas feitas a mão em laboratório, através da tela de formação.

Em virtude da variação da gramatura com os tratamentos de refinação da celulose, os resultados médios das resistências mecânicas do papel são apresentados como índices de rasgo e estouro e, como comprimento de auto-ruptura, para comparação dos resultados, pois nos cálculos desses índices elimina-se o efeito da gramatura, conforme afirma Sanjuan (10).

A densidade aparente do papel feito a mão, demonstra como as fibras (traqueóides) se compactaram pelo efeito dos tratamentos de refinação, elevando os resultados com o aumento do tempo de refinação, este comportamento é esperado, de acordo com IPT (11) e Sanjuan (10).

A tabela 4 apresenta o resumo dos testes de médias (" $t$ " de Student) realizados para comparação dos valores médios obtidos entre as espécies nos tratamentos de refinação. 
Tabela 4: Resumos dos testes de média, para as propriedades de resistência das folhas de papel feitas a mão, a partir de celulose Kraft da madeira juvenil de Pinus maximinoi e de Pinus taeda, entre tratamentos de refinação

Table 4: $\quad$ Mean tests for resistance properties from paper handsheets obtained from Kraft pulps of juvenile wood from Pinus maximinoi and Pinus taeda between refinning treatments

\begin{tabular}{|c|c|c|c|c|}
\hline Propriedade & Tratamento & Pinus maximinoi & Pinus taeda & Avaliação \\
\hline Índice de rasgo & Sem refinação & 22,96 & 23,96 & n.s \\
\hline Índice de estouro & Sem refinação & 1,83 & 2,13 & $* *$ \\
\hline Densidade aparente & Sem refinação & 0,42 & 0,39 & $* *$ \\
\hline Índice de rasgo & $20 \mathrm{~min}$. & 19,58 & 19,76 & n.s \\
\hline Comprimento de auto-ruptura & $20 \mathrm{~min}$. & 6,05 & 5,96 & n.s. \\
\hline Densidade aparente & $20 \mathrm{~min}$ & 0,59 & 0,59 & n.s. \\
\hline Índice de rasgo & $40 \mathrm{~min}$ & 13,68 & 15,88 & $* *$ \\
\hline Índice de estouro & $40 \mathrm{~min}$. & 4,22 & 4,20 & n.s. \\
\hline Índice de estouro & $60 \mathrm{~min}$ & 4,23 & 4,00 & $* *$ \\
\hline Comprimento de auto-ruptura & $60 \mathrm{~min}$. & 6,98 & 6,47 & $* *$ \\
\hline Densidade aparente & $60 \mathrm{~min}$. & 0,69 & 0,66 & n.s. \\
\hline
\end{tabular}

n.s $=$ não significativo $* *=$ significativo a $99 \%$ de probabilidade

Legenda:

Índice de rasgo $\left(\mathrm{mN} \cdot \mathrm{m}^{2} \cdot \mathrm{g}^{-1}\right)$

Índice de estouro $\left(\mathrm{kPa} \cdot \mathrm{m}^{2} \cdot \mathrm{g}^{-1}\right)$

Comprimento auto-ruptura $(\mathrm{km})$

Densidade aparente (g. $\left.\mathrm{cm}^{-3}\right)$

Analisando os resultados apresentados nas tabelas 3 e 4 e na figura 1, observa-se a tendência de decréscimo dos índices de rasgo com o aumento do tempo de refinação para as espécies, o que é esperado em razão da ação do tratamento mecânico de desfibrilamento das paredes celulares, as diferenças entre os tratamentos são estatisticamente significativas para as duas espécies.
A tabela 5, apresenta o resumo dos testes de médias (" $t$ " de Student) realizados para comparação dos valores médios gerais das propriedades entre as espécies, e a tabela 6 , apresenta os resumos das análises de variância para verificação das diferenças entre os tratamentos de refinação.

Tabela 5: Resumos dos testes de média para as propriedades médias da celulose Kraft e das médias gerais dos tratamentos de refinação das folhas de papel feitas a mão, da madeira juvenil, entre Pinus maximinoi e de Pinus taeda

Table 5: $\quad$ Mean tests for average properties from Kraft paper handsheets and from beating treatments average from Kraft hand made sheets paper of juvenile wood between Pinus maximinoi and Pinus taeda

\begin{tabular}{lccc}
\hline \multicolumn{1}{c}{ Característica/ Propriedade } & Pinus maximinoi & Pinus taeda & \\
\hline Rendimento bruto (\%) & 45,26 & 45,76 & n.s \\
Teor de rejeitos (\%) & 0,20 & 0,81 & $* *$ \\
Número Kappa & 31,74 & 32,56 & n.s. \\
Índice de rasgo $\left(\mathrm{mN}^{2} \mathrm{~m}^{2} . \mathrm{g}-1\right)$ & 16,86 & 17,98 & n.s. \\
Índice de estouro $\left(\mathrm{kPa} \cdot \mathrm{m}^{2} . \mathrm{g}-1\right)$ & 3,51 & 3,58 & n.s. \\
Comprimento auto-ruptura $(\mathrm{km})$ & 5,77 & 5,62 & n.s \\
Densidade aparente $\left(\mathrm{g} \cdot \mathrm{cm}^{-3}\right)$ & 0,58 & 0,57 & n.s \\
\hline
\end{tabular}

n.s. = não significativo $* *=$ significativo a $99 \%$ de probabilidade 
Tabela 6: Resumo das análises de variância para as propriedades mecânicas do papel feito a mão da madeira juvenil de Pinus maximinoi e de Pinus taeda entre os tratamentos de refinação

Table 6: Variance analysis between beating treatments for properties from Kraft paper handsheets paper of Pinus maximinoi and Pinus taeda juvenile wood

\begin{tabular}{lccc}
\hline \multicolumn{1}{c}{ Propriedade } & $\begin{array}{c}\text { Quadrado Médio } \\
\text { Tratamentos }\end{array}$ & Quadrado Médio Erro & F (3,40) \\
\hline Índice de rasgo & 33511 & 481,12 & 69,652 \\
Índice de estouro & 1389 & 0,34 & 4145,688 \\
Comprimento auto-ruptura & 29 & 0,017 & $* *$ \\
Densidade aparente & 0,1 & 0,0001 & 2075,378 \\
\hline
\end{tabular}

** = significativo a $99 \%$ de probabilidade

Os valores médios dos índices de rasgo foram ligeiramente superiores para a celulose de Pinus taeda, porém na média geral dos tratamentos a diferença existente não foi significativa enquanto que, nos tratamentos observa-se que apenas a 40 minutos de refinação ocorre diferença estatística significativa para o índice de rasgo, que é inferior para a celulose de Pinus maximinoi, o que pode estar relacionado a maior desfibrilação das paredes das fibras de $P$. maximinoi, em razão da madeira apresentar menor porcentagem de lenho tardio comparado a $P$. taeda da mesma idade e condição de crescimento, Klock (12).

$\mathrm{O}$ índice de estouro, ao contrário da resistência ao rasgo, é incrementado com os tratamentos de refinação dos traqueóides, em função do desfibrilamento das paredes celulares que ocorre no processo, provocando maior hidratação e melhor conformação das fibras nas folhas de papel formadas, incrementa as ligações hidrostáticas entre as fibrilas de celulose com o aumento do tempo de refinação até certo grau. As diferenças entre os tratamentos, como na resistência ao rasgo são estatisticamente significativas. Entre as espécies observa-se no tratamento sem refinação e a 20 minutos de refinação diferenças estatisticamente significativas, sendo os resultados médios inferiores para $P$. maximinoi, já a 40 minutos de refinação os valores médios foram similares, invertendo-se os valores médios a 60 minutos de refinação, onde o índice de estouro foi superior para a celulose de $P$. maximinoi, com diferença significativa, observou-se também um decréscimo do índice de estouro no papel de $P$. taeda entre 40 e 60 minutos de refinação, o que pode indicar que o máximo de resistência foi atingido nesse intervalo, enquanto que as fibras de $P$. maximinoi apresentaram melhor resistência.
Como pode ser observado na figura 2 a resistência ao estouro para a celulose de Pinus maximinoi desenvolveu-se mais que a de Pinus taeda nos tratamentos de refinação testados. De valor inicial menor, ultrapassou a resistência ao estouro de Pinus taeda aos 60 minutos de refinação. Entretanto, as médias gerais dos tratamentos entre as espécies não diferem estatisticamente, ocorrendo porém diferenças significativas no desenvolvimento da resistência ao estouro, durante o processo de refinação da polpa celulósica.

O comprimento médio de auto-ruptura, assim como o índice de estouro, foi incrementado com os tempos dos tratamentos de refinação, até um certo limite, o que também aconteceu em função do desfibrilamento nas camadas da parede celular. Os aumentos foram superiores entre o tratamento sem refinação e até 20 minutos de refinação, as diferenças entre os valores médios dos tratamentos são estatisticamente significativas, como também ocorreu para as resistências ao rasgo e ao estouro, confirmando o esperado efeito da refinação sobre as propriedades das fibras e do papel.

Observa-se que no tratamento sem refinação o valor médio do comprimento de auto-ruptura do papel de Pinus maximinoi foi $11,5 \%$ inferior ao de Pinus taeda, diferença estatisticamente significativa, sendo amplificada com o aumento dos tempos de refinação, com 20 minutos foi ligeiramente superior não deferindo, já aos 40 minutos a diferença foi $6,6 \%$ superior, chegando a $7,9 \%$ aos 60 minutos de refinação, ambas significativas estatisticamente (tabela 4). Notase que, como ocorreu com a resistência ao estouro, a resistência à tração do papel de Pinus maximinoi desenvolveu-se de forma mais acentuada com os tratamentos de refinação aplicados. 
O comprimento de auto-ruptura, que é uma das mais importantes e estudadas propriedades mecânicas do papel, apresenta alta correlação com as características da madeira e ocorrem de forma negativa com a densidade, com a porcentagem de lenho tardio e também com a espessura média da parede celular, Dinwoodie (13) e Foelkel (5). Considerando-se porém, que outros fatores podem também influir nas propriedades de tração do papel, em função das espécies utilizadas, como as características das fibras e outros aspectos, é importante analisar as características das duas espécies em estudo e correlaciona-las.

A densidade aparente do papel aumenta com o tempo de refinação, também em função do desfribilamento das paredes celulares, o que permite uma melhor acomodação das fibras no papel, permitindo ainda uma melhor compactação destas nas folhas, de tal forma que a maiores tempos de refinação ter-se-á papel com maior densidade aparente, Sanjuan (10).

As diferenças observadas nesse estudo concordam com essa tendência e são estatisticamente significativas entre os tratamentos de refinação para as duas espécies estudadas. Os resultados médios por tratamento de refinação entre as espécies mostram tendência semelhante entre ambas, ocorrendo entretanto, no tratamento sem refinação maior densidade aparente para o papel de Pinus maximinoi. Porém, com a aplicação dos tratamentos de refinação, o comportamento da densidade aparente para a celulose das duas espécies torna-se similar, indicando uma densidade aparente semelhante para o papel das duas espécies.

Entre os resultados desse estudo e os relatados por Wright \& Wessels (2), nota-se que

os índices de rasgo foram superiores, enquanto que os valores para a variação do índice de estouro e comprimento de autoruptura foram inferiores, estas diferenças podem estar relacionadas as diferenças entre os procedimentos de obtenção da celulose, indicado pelos diferentes números Kappa, 31 e 55 respectivamente, das diferenças nas condições de refinação, como do equipamento utilizado, e também nas condições de crescimento das árvores.
Os resultados nesse estudo assemelham-se aos relatados por Watson \& Dadswell (6) considerando-se o tratamento sem refinação e sendo inferiores no tratamento com refinação, especialmente em rasgo e estouro, o que possivelmente está relacionado à maior idade do material utilizado naquele estudo, em razão das diferenças na proporção de madeira adulta e conseqüente diferenciação dos traqueóides.

Já ao se considerar os resultados apresentados por Klock et al. (7), os valores superiores obtidos, como mencionado anteriormente, devem estar relacionados a maior idade das árvores. Porém o comportamento dos resultados com o tempo de refinação deste estudo assemelha-se ao observado pelos autores.

\section{CONCLUSÕES E RECOMENDAÇÕES}

As características da celulose obtida da madeira de Pinus maximinoi mostram rendimento esperado para a madeira de árvores de rápido crescimento de espécies do gênero Pinus, sendo equivalente ao de Pinus taeda na mesma idade e condições de cozimento, apresentando boas características de deslignificação com baixo teor de rejeitos.

O papel feito à mão a partir da celulose Kraft de Pinus maximinoi apresentou, em média, qualidade semelhante à de Pinus taeda, indicando sua potencialidade para essa utilização. As propriedades de resistência do papel feito a mão da madeira de Pinus maximinoi evoluíram nos tratamentos de refinação de forma mais homogênea, quando comparadas às mesmas propriedades obtidas com madeira de Pinus taeda.

Conclui-se que a madeira de Pinus maximinoi apresenta características tecnológicas sua utilização para a fabricação de celulose e papel.

Recomenda-se analisar as características das duas espécies estudadas e correlaciona-las.

\section{AGRADECIMENTOS}

A NORSKE SKOG - Papel de Imprensa $\mathrm{S}$. A., pela cessão do material para pesquisa. 
A Universidade Federal do Paraná Departamento de Engenharia e Tecnologia Florestal, pelo apoio e realização do trabalho.

Obs: Trabalho apresentado no Congreso Iberoamericano de Investigación em Celulosa y Papel 2002 na forma de poster.

\section{REFERÊNCIAS}

BRACELPA, Estatísticas do Setor Disponível: http://www.bracelpa.org.br/, Capturado em 09/2004.

WRIGHT, J. A., WESSELS, A. "Laboratory scale pulping of Pinus pseudostrobus, $P$. maximinoi and $P$. patula". IPEF International, Piracicaba, n.2, p.39-44, 1992.

WRIGHT, J. A \& BAYLIS, B. Volume, pulp and paper-making traits of Pinus maximinoi provenances planted at two sites in South Africa. South African Forestry Journal. n.165, p.37-40, 1993.

WRIGHT, J. A; JAMEEL, H; DVORAK, W. "Laboratory Kraft pulping of juvenile tropical pines: Pinus patula, $P$. tecunumanii, $P$. maximinoi and $P$. chiapensis". Tappi Journal, v.79, n.4, p.187-190, 1996.

FOELKEL, C. E. B. Celulose Kraft de Pinus spp. O Papel. ABCP, São Paulo, p.49-67. 1976.

WATSON, A. J. \& DADSWELL, H. E. "Influence of fibre morphology on paper properties - Part 1. Fibre length" .APPITA. v.14, n.5, p.168-178. 1961.
KLOCK, U. SIMÃO, R. L., MENDES, F. BITTENCOURT, E.; ANDRADE, A S. "Massa específica básica, porcentagem de lenho tardio ao longo do fuste, características dos traqueóides, composição química quantitativa e propriedades das fibras em papel feito a mão de Pinus taeda L". Relatório Técnico. FUPEF. 2001.

ABTCP - ASSOCIAÇÃO BRASILEIRA TÉCNICA DE CELULOSE E PAPEL. Pasta Celulósica e Papel. São Paulo. 1998.

SMOOK, G. A. Handbook for pulp and paper technologists. Joint Textbook. Committee of the Paper Industry. TAPPI, Atlanta, 1989.

SANJUAN, R. D. "Obtención de pulpas y propiedades de las fibras para papel". Guadalajara: Universidad de Guadalajara, 1997. 293p.

IPT. Celulose e papel. São Paulo: vol I, $2^{\circ}$ ed. Instituto de Pesquisa Tecnológica de São Paulo. 1988.

KLOCK, U. "Qualidade da madeira juvenil de Pinus maximinoi H. E. Moore". Curitiba: 2000. 291p. Tese. (Doutorado em Engenharia Florestal) Curso de Pós-graduação em Engenharia Florestal. Universidade Federal do Paraná.

DINWOODIE, J. M. "The relationship between fiber morphology and paper properties: A review of literature". Tappi. V. 48, n.8. p.440-446. 1965. 
Propriedades do papel Kraft... 